\title{
Empreinte parentale du génome et cancers congénitaux
}

Il existe des formes héréditaires et des formes sporadiques des cancers congénitaux du type rétinoblastome et néphroblastome [1]. Dans les premières, l'enfant hérite de l'un de ses parents un «gène de susceptibilité » anormal.

Un événement somatique aboutit alors à la perte de l'allèle issu du parent sain, ce qui provoque le développement de la tumeur. Dans les formes sporadiques, le premier événement survient soit au niveau d'un gamète mâle ou femelle (il sera alors à l'origine d'une forme héréditaire), soit d'une cellule somatique. Le second événement, dans les formes sporadiques aussi bien qu'héréditaires, est le plus souvent une recombinaison mitotique, ou une non-disjonction chromosomique, et aboutit à la perte de tout ou partie du chromosome portant l'allèle sain.

Différentes équipes européennes, japonaises et américaines ont en fait démontré que, dans ces formes sporadiques, c'était le chromosome paternel qui était retrouvé dans les tumeurs (néphroblastomes ou rétinoblastomes, ostéosarcomes), l'allèle maternel étant perdu [2, 5]. Le taux de mutation étant probablement différent dans les gamètes mâles et femelles, les auteurs ont exclu de leurs études toutes les observations où le premier événement pouvait être germinal ; malgré cela, neuf cas d'ostéosarcome avec anomalie du locus $\mathrm{Rb}$ (gène de susceptibilité au rétinoblastome en 13q14) sur 10 [5] et cinq cas de néphroblastome avec anomalie de la région $1 \mathrm{lpl} 3$ (censée conte- néphroblastome) sur 5 [4] possédaient l'allèle paternel, et non l'allèle maternel. Les résultats sont interprétés en invoquant une «empreinte » parentale sur les deux allèles, l'empreinte paternelle prédisposant les gènes de susceptibilité à une mutation inactivante [3-6].

La base moléculaire de l'« empreinte parentale » est probablement une différence de méthylation de dinucléotides CG ; une méthylation des cytosines favorise leur désamination en thymine. Une hyperméthylation (non prouvée) des allèles paternels pourrait donc favoriser une telle sensibilité aux mutations. Une autre possibilité serait que des mutations, survenant sinon à la même fréquence au niveau des allèles paternels et maternels, confèrent un avantage spécifique aux cellules possédant un allèle paternel muté. Un semblable scénario serait possible si c'étaient, en fait, les allèles maternels qui étaient, dans le plus grand nombre des cellules de l'embryon, hyperméthylés et donc inactifs. Une mutation d'un allèle maternel, ou d'un allèle paternel coexistant avec un allèle maternel déméthylé (c'est-à-dire actif), n'aurait pas de conséquence car persisterait, dans la cellule, un anti-oncogène (c'est-à-dire l'un des deux gènes de susceptibilité) actif. En revanche, la cellule ayant un allèle paternel muté et un allèle maternel inactif car hyperméthylé ne produirait pas la protéine anti-oncogénique codée par le gène de susceptibilité et subirait une expansion clonale qui pourrait cesser lors de la déméthylation de l'allèle sain ou être à l'origine d'une tumeur irreversible en cas de perte de cet allèle. Il faut remarquer que ce dernier événement aurait alors d'autant plus de risque de se produire que la population, déjà hétérozygote pour la mutation, serait, du fait de son expansion, devenue abondante [6].

L'un des gènes de susceptibilité (celui du rétinoblastome) étant maintenant connu, ces différentes hypothèses devraient pouvoir être directement testées. Des études ultérieures diront aussi si de tels phénomènes peuvent être étendus aux autres cancers dans la genèse desquels semble intervenir une perte de matériel génétique et une inactivation d'anti-oncogènes.

A.K.

1. Junien C. Les anti-oncogènes. médecine/ sciences $1986 ; 2$ : 238-45.

2. Junien C, Henry L. Empreinte parentale différentielle, tumorigenèse et perte d'allèles. Nouvelles interprétations. médecine/sciences 1989 (sous presse).

3. Mannens M, Slater RM, Heyting C, et al. Molecular nature of genetic changes resulting in loss of heterozygosity of chromosome 11 in Wilms tumours. Hum Genet 1988 ; 81 : 41-8.

4. Schroeder WT, Chao LY, Dao DD, et al.. Nonrandom loss of maternal chromosome 11 alleles in Wilms tumours. Am J Hum Genet 1987 ; 40 : 413-30.

5. Toguchida J, Ishizaki K, Sasaki M, et al. Preferential mutation of paternally derived $R B a$ gene as the initial event in sporadic osteosarcoma. Nature $1989 ; 338$ : 156-8.

6. Reik W, Surani MA. Genomic imprinting and embryonal tumours. Nature $1989 ; 338$ : 112-3. 